\title{
MBBR Technology for Nutrient Removal at Lagoon Facilities: Cold Climate Performance and Bacterial Community Analysis
}

\author{
Robert Delatolla \\ Civil Engineering Department, University of Ottawa \\ Canada
}

The challenges of achieving nutrient removal at passive treatment facilities such as lagoons include the availability of proven, economical and low operational intensity technologies to operate as upgrade units that supplement the current infrastructure of the passive treatment systems. Furthermore, temperate and cold climate countries such as Canada face the additional challenges of operating these upgrade technologies at the low wastewater temperatures characteristic of lagoon effluent during winter months; with nitrification rates being shown to decline substantially or become completely impeded during winter operation. This research investigates the use of the moving bed biofilm reactor (MBBR) technology as a low operational intensity upgrade solution to lagoon facilities in Canada to not only achieve year-round, cold temperature nitrification but to also achieve significant total nitrogen removal via conventional nitrification/denitrification, and to achieve total phosphorus removal via biomass assimilation. The study moves beyond a demonstration of performance and kinetic analyses attained at the pilot scale and uses advanced microscopy and molecular methods, such as variable pressure scanning electron microscopy (VPSEM), confocal laser scanning microscopy (CLSM) in combination with viability staining along with Illumina next-generation sequencing, to develop a fundamental understanding of nutrient removal biofilms in the MBBR technology applied as lagoon upgrade units. 